\title{
Laparoscopic Heller Esophagomyotomy is Safe and Effective in Rural East Africa
}

\author{
Wairimu Ndegwa, ${ }^{1}$ Hill Katherine, ${ }^{1,2}$ Parker Robert, ${ }^{1,3}$ White Russell, ${ }^{1,3}$ Many Heath ${ }^{1,4}$ \\ 1 Tenwek Mission Hospital, Bomet \\ 2 University of Pittsburgh Medical Center, Pittsburg, California \\ ${ }^{3}$ Brown University, Providence, Rhode Island \\ ${ }^{4}$ University of Tennessee, Knoxville, Tennessee
}

Correspondence to: Dr. Wairimu Ndegwa, PO Box 39-20400 Bomet, Kenya; email: ndegwa.wairimu@gmail.com

\begin{abstract}
Background: The incidence of achalasia in subSaharan Africa is not known. Experience in our region suggests the disorder affects mainly younger patients. Esophagomyotomy is the gold standard treatment for achalasia. Benefits of laparoscopic treatment are well documented in western populations. African data is insufficient. Methods: A retrospective review of patients over 16 years who underwent esophagomyotomy at Tenwek Hospital (2008-2017). The primary outcome was improvement in symptoms before and after surgery as measured using the Eckardt score- lower scores for dysphagia, regurgitation, pain, weight loss indicate less severe symptoms. The secondary outcomes were duration of surgery, length of myotomy, length of hospital stay, and complication rate. Data analysis used ANOVA. Results: 54 patients were included: 28 with laparoscopic Heller myotomy (LHM), 26 with open Heller myotomy (OHM). LHM patients were younger than OHM patients $(\mathrm{p}<0.05)$. Patients who had LHM
\end{abstract}

had lower postoperative Eckardt scores $(\mathrm{p}<0.05)$. Duration of surgery for LHM was longer $(p<0.001)$ than for OHM, conversion rate was $10.71 \%$, and length of the myotomy was unaltered. No difference was seen in hospital stay or complication rate between the two groups. Conclusion: LHM is effective and safe in a rural East African setting, with excellent functional outcomes compared with open techniques. Thus, laparoscopy can be feasible worldwide.

Keywords: Laparoscopic, Heller Esophagomyotomy, Tenwek Hospital, Achalasia

Ann Afr Surg. 2020; 17(1):21-25

DOI: http://dx.doi.org/10.4314/aas.v17i1.6

Conflicts of Interest: None

Funding: None

(C) 2020 Author. This work is licensed under the Creative Commons Attribution 4.0 International License.

\section{Introduction}

Esophagomyotomy (Heller myotomy) is described as the gold standard treatment for esophageal achalasia $(1,2)$. Achalasia is a neurodegenerative motility disorder of the esophagus, resulting in deranged peristalsis and loss of lower esophageal sphincter function. The etiology is unclear but likely to be multifactorial (3). Treatment of achalasia involves myotomy and has been commonly managed with laparoscopic Heller myotomy (LHM). Achalasia is a rare primary esophageal motility disorder that occurs with equal distribution, irrespective of gender and race. The incidence of achalasia in western populations is as low as $0.5-1 / 100,000$ people and accounts for $3-20 \%$ of cases among esophageal diseases (4). The incidence of achalasia in sub-Saharan Africa has not been established; a few studies exist, mainly within the northern and western Africa regions $(5,6)$, but

studies are lacking in East and Central Africa. Therefore, we sought to evaluate the efficacy and safety of LHM in our population. We undertook this 9-year retrospective review to compare LHM with open Heller myotomy (OHM).

\section{Materials and Methods}

\section{Patients, setting, and surgical technique}

The study was conducted at Tenwek Hospital, a rural hospital in western Kenya in the south rift region. The research and ethics review committee at Tenwek Hospital gave approval to proceed with the study, after which all patients who were included in the study were informed and gave written consent for use of any data and photos.

We reviewed retrospectively records of all patients who had a diagnosis of achalasia and who underwent LHM or 
OHM between 2008 and 2017. The age of these patients in the series was 16-74 years. For each patient included, the diagnosis had been established by barium swallow esophagram; an endoscopic evaluation excluded other pathology. Exclusion criteria included: 1 ) age $<16$ - the focus was

Table 1. The Eckardt score: final score is the sum of the 4 components

\begin{tabular}{clllc}
\hline Score & Dysphagia & Regurgitation & $\begin{array}{l}\text { Retrosternal } \\
\text { pain }\end{array}$ & $\begin{array}{l}\text { Weight loss } \\
(\mathrm{kg})\end{array}$ \\
\hline 0 & None & None & None & None \\
1 & Occasional & Occasional & Occasional & $<5$ \\
2 & Daily & Daily & Daily & $5-10$ \\
3 & Each meal & Each meal & Each meal & $>10$ \\
\hline
\end{tabular}
on adult patients, and in the data collected no paediatric patient files were found with a diagnosis of achalasia, 2) patients who underwent endoscopic dilation as therapy for achalasia before myotomy.

For the procedure, the patient is under general anaesthesia in low lithotomy position with the surgeon positioned caudal and the assistants on the right and left of the patient. The patient's abdomen is insufflated with carbon dioxide, and laparoscopic instruments are introduced via five keyhole incisions $(1-2 \mathrm{~cm})$ made on the abdomen. The lower oesophagus was approached and a lengthwise cut was made in the muscle layer. Care was taken to cut only the muscle layer, leaving the inner lining of the oesophagus intact. As this procedure is often complicated by acid reflux after the operation, a partial or complete fundoplication is also performed at the same time to minimize this. The Dor partial fundoplication was the anti-reflux procedure performed for all patients. Participating surgeons prefer this method to avoid the distal esophagus angulating, and because it requires less extensive dissection than total fundoplication $(7,8)$. Most participating surgeons routinely use intraoperative esophagogastroduodenoscopy (EGD) during LHM, after completion of the myotomy.

\section{Outcome measures}

The primary outcome was improvement in pre- to postoperative symptoms, measured by Eckardt Symptom Score (ESS). The ESS is the grading system most frequently used to evaluate symptoms and stages of achalasia and efficacy of treatment. It attributes points from 0 to 4 , based on the symptoms of the disease (dysphagia, regurgitation, chest pain, and weight loss), giving a cumulative score that ranges from 0 to 12 (Table $1)$. Scores of $0-1$ correspond to clinical stage $0,2-3$ to stage I, 4-6 to stage II, and a score $>6$ to stage III (Table 2) $(9,10)$. Secondary outcomes included duration of surgery, length of myotomy, hospital length of stay, and complication rate. Possible complications included mucosal perforations, inadvertent pleural cavity breech, unplanned take back. Data on intraoperative revision of the myotomy post-EGD were not available for analysis.

\section{Data collection}

Patient charts from 2008-2014 were reviewed vide

Table 2. Clinical scoring system for achalasia (16)

\begin{tabular}{ccl}
\hline Stage & Eckardt score & Clinical implication posttreatment \\
\hline 0 & $0-1$ & Remission \\
I & $2-3$ & Remission \\
II & $4-6$ & Treatment failure \\
III & $>6$ & Treatment failure \\
\hline
\end{tabular}

paper medical records while those from 2014-2017 were accessed vide the hospital electronic medical record system. Files of patients who underwent esophagomyotomy for a diagnosis of achalasia between 2008 and 2017 were retrieved and reviewed. Patients who were 16 years and below were excluded as were patients who had undergone endoscopic dilation as therapy for achalasia before myotomy. Patients had been evaluated in the surgical outpatient clinic; they completed a questionnaire based on the ESS to evaluate the current symptoms they were experiencing. In our setup, data were collected with the help of clinical translators who had the questionnaire explained to them beforehand, and data were entered in real time at the time of the interviews. The surgeon selected the patients; selection criteria was based on the availability and safe functionality of the laparoscopy equipment. Patients who did not undergo the procedure were scheduled for open Heller esophagomyotomy.

Preoperative measurements consisted of identifying common symptoms associated with the disorder and then quantifying them as scores. Scoring used a self-designed questionnaire. Symptoms were scored according to their frequency of dysphagia, regurgitation and retrosternal pain, and quantity of weight lost based on a score of 0 to 3 , to give a possible cumulative total of between 0 and 12 (Tables 1 and 2). The result was the Eckardt score. As an example, a patient with occasional dysphagia, daily regurgitation, occasional retrosternal pain and with $6 \mathrm{~kg}$ weight loss would have an Eckardt score of 6.

Postoperative symptomatology was assessed using the same parameters to calculate posttreatment Eckardt score and correlate this score with the clinical. Standardized questionnaires were filled in during clinic 
visits or through a telephone interview with the patients. Questionnaires were filled between 6 and 24 months postoperative. The questionnaires also contained information about preoperative investigations, i.e. CXR, barium swallows and CT scans.

\section{Statistical analysis}

Groups were compared using chi-square for categorical data, and Student $t$-test and Mann-Whitney test for continuous data. ANOVA was used to analyse the interaction between use of intraoperative EGD and postoperative ESS.

\section{Results}

Fifty-four patients met the inclusion criteria: 28 had undergone LHM and 26 OHM. Table 1 shows descriptive statistics comparing the two groups. Patients who had LHM were younger than those with OHM $(\mathrm{p}<0.05)$, with no significant difference in pre-op ESS or duration of symptoms.

The $\mathrm{t}$ mean preoperative ESS for the total patient population was 7.14 , indicating symptoms as being clinical stage III (Table 2) (16). Of the four score components, the most common presenting complaint was dysphagia seen in $37.1 \%$, next was weight loss at $25 \%$, pain was experienced by $21.4 \%$ and regurgitation by $17.9 \%$. The presence of regurgitation seemed to be a late sign, indicating advanced disease, whereas dysphagia was the earliest symptom, causing the greatest effect on patient quality of life.

\section{Primary outcome}

Among all patients, 54(93\%) experienced lower postoperative ESS, and thus improvement in symptoms. Patients who had had LHM had lower postoperative ESS $(p<0.05$, Table 3$)$ than those who had an OHM.

With no difference between groups in preoperative ESS, the indication is a possible greater symptomatic improvement among those undergoing LHM.

The use of intraoperative EGD was disproportionately represented among the LHM group (OR 36.82, 95\% CI, 4.32-313.63 for LHM), due to its inclusion as a standard part of this operative technique. When controlling for intraoperative EGD, postoperative ESS no longer demonstrates significant differences between groups $(\mathrm{p}=0.4)$.

\section{Secondary outcomes}

Duration of surgery for LHM was longer than for OHM $(\mathrm{p}<0.001)$ with a $10.71 \%$ conversion rate, but length of myotomy did not differ. No statistically significant difference was observed in hospital length of stay, with LHM being slightly shorter than OHM, or in complication rate, which was $7.14 \%$ overall. No mortalities or re-admissions were reported. Table 4 shows these results. Complications included two mucosal perforations, an inadvertent pleural cavity breech, and an unplanned take back. Blood loss for the procedure was approximately $10 \mathrm{cc}$.

\section{Discussion}

In treating achalasia, the focus is on alleviating symptoms rather than on addressing the etiology-a neurodegenerative pathology that is irreversible. The most common primary symptom on presentation remains dysphagia, next are pain and regurgitation (9). The frequency of symptoms in our patient population was consistent with that in north and west Africa where similar studies have been conducted $(5,6)$. Compared with data in industrialized countries, the frequency of symptoms was also comparable $(2,3,11,15)$. The ESS is a fair measure of achalasia symptom severity; its strengths are in assessing dysphagia and regurgitation, factors that consistently performed well when assessed for reliability and validity. The apparent weaknesses of the ESS lie in the chest pain, due to the heterogeneous nature of chest pain, and weight loss, because weight loss may be multifactorial in origin (9).

Our study shows that Heller myotomy affords significant relief of the symptoms of dysphagia, regurgitation and chest pain. Total symptom scores improved for 
all patients, a success rate that agrees with published literature (7). With the significant improvement on patient symptoms after LHM, we can infer a better quality of life, as described by Decker et al. (11).

This single-centre retrospective review provides a unique analysis of surgical treatment of achalasia in a rural African setting with resource limitation. The patient population of this study is also different from that presented in western data, with a higher likelihood of advanced pathology such as sigmoid esophagus than in the western population and coupled with limitations in diagnostic capabilities (lack of manometry) introduces the potential for poor outcomes.

The challenges of carrying out laparoscopy in a rural setting extend beyond just having the right equipment. Issues encountered in our setting include unreliable electricity, lack of operative support personnel to assist in troubleshooting problems that occur intraoperatively, supply chain issues, and patients who have limited resources to fund the procedure and to allow appropriate follow-up. While some of these factors may not affect the primary outcome substantially, they certainly could be reflected in the complication rate and the need to convert to open surgery. A combination of these factors may even render the procedure unfeasible in our setting. These factors can also have a significant impact on cost and further implementation of the procedure.

While patients undergoing LHM were significantly younger than those with OHM, the mean age for both cohorts is dramatically younger than in previously published data on western populations (12). This younger age reflects a possible difference in disease aetiology, population characteristics or risk factors and warrants further study.

With the advent of minimally invasive procedures, reduced complication rates and decreased hospital length of stay have been demonstrated among primarily western patient populations (7). In this study, we aimed to demonstrate that LHM does not have inferior outcomes to OHM, which has been carried out for longer in most parts of Africa and indeed in Kenya. Alleviating patient symptoms may be greater with LHM, which showed a trend toward shorter hospital length of stay.

It is not surprising that the duration of surgery was longer for the laparoscopic cases, which we can attribute to the challenges faced in a resource-limited environment coupled with the procedure's steep learning curve.

The surgeons who performed the procedures had previous experience in a western setting, and a true learning curve that accompanies a de novo introduction of LHM would not be reflected in this study. Laparoscopy was introduced at the facility in 2009 and its use gradually increased to become the main treatment modality for achalasia.

Previously published work notes that this learning curve typically plateaus after 20 esophagomyotomies, at which point the surgeon has accumulated sufficient experience and confidence (13). Anecdotally, technical issues that may have contributed to duration of the procedure included frequent malfunction of the equipment with difficulty in troubleshooting, thus prolonging operative time. Our conversion rate of $10.71 \%$ and low complication rate agree with other literature, indicating the feasibility of LHM in our setting $(1,14)$.

The limitations of this study include its retrospective nature, particularly in defining preoperative Eckardt scores from data in patient charts. In addition, variability in the level of proficiency of operating surgeons was not evaluated in this study. Within a teaching institution such as a rural hospital in Kenya, the case could be performed by an attending surgeon or by a surgical resident under the supervision of the attending surgeon; however, which individual performed critical portions of the case is not routinely recorded in the medical record. Patient follow-up was poor as most of the patients would not attend follow-up clinics in the recommended time post-procedure. Some did not provide a telephone contact which in turn had the effect of prolonging the time to complete the questionnaire. An additional limitation is the lack of documentation on the choice of laparoscopy versus an open procedure. The initial thought was that this decision was based on the availability of laparoscopy services during the study period (i.e. availability of working equipment and $\mathrm{CO}_{2}$ ). However, a notable difference in baseline characteristics was that older patients were more likely to undergo an open procedure, owing to patient or surgeon preference, but this warrants further investigation. Our analyses were limited by our small sample size, so while the study provides an initial snapshot of LHM within an East African population, further studies with larger samples sizes would be beneficial. A prospective arm of study is being considered to evaluate the changes, if any, that have been brought about by introducing laparoscopy in our setting.

\section{Conclusion}

We demonstrate that laparoscopic Heller esophagomyotomy is effective and safe in a rural East African setting, with excellent functional outcomes compared with open 
techniques, despite several limitations ranging from limited availability of advanced diagnostic modalities (manometry), advanced patho-logy at presentation and lack of funds (patient) to sub-optimal patient followup. Our findings contribute to growing evidence that laparoscopy can be feasible worldwide.

\section{References}

1. Campos G, Vittinghoff E, Rabl C, et al. Endoscopic and surgical treatments for achalasia: A systematic review and meta-analysis. Ann Surg. 2009; 249(1):45-57.

2. Wang L, Li YM, Li L, et al. A systematic review and metaanalysis of the Chinese literature for the treatment of achalasia. World J Gastroenterol. 2008; 14(38):5900-6.

3. O’Neill OM, Johnston BT, Coleman HG. Achalasia: A review of clinical diagnosis, epidemiology, treatment and outcomes. World J Gastroenterol. 2013; 19(35):5806-12.

4. Mayberry JF. Epidemiology and demographics of achalasia. Gastrointest Endosc Clin N Am. 2001; 11(2):235-48.

5. Ahmed A, Yusufu LM, Ukwenya YA, et al. Surgical management of achalasia in Zaria, Northern Nigeria. S Afr J Surg. 2008; 46(2):48-51.

6. Tebaibia A, Boudjella MA, Boutarene D, et al. Incidence, clinical features and para-clinical findings of achalasia in Algeria: Experience of 25 years. World J Gastroenterol. 2016; 22(38):8615-23.

7. Sharp K, Khaitan L, Scholz S, et al. 100 consecutive minimally invasive Heller myotomies: Lessons learned. Ann Surg. 2002; 235(5):631-8.

8. Katilius M, Velanovich V. Heller myotomy for achalasia:
Quality of life comparison of laparoscopic and open approaches. JSLS. 2001; 5(3):227-31.

9. Taft T, Carlson D, Triggs J, et al. Evaluating the reliability and construct validity of the Eckardt symptom score as a measure of achalasia severity. Neurogastroenterol Motil. 2018; 30(6): e13287.

10. Eckardt A, Eckardt V. Treatment and surveillance strategies in achalasia: An update. Nat Rev Gastroenterol Hepatol. $2011 ; 8(6): 311-9$.

11. Decker G, Borie F, Bouamrirene D, et al. Gastrointestinal quality of life before and after laparoscopic Heller myotomy with partial posterior fundoplication. Ann Surg. 2002; 236(6):750-8.

12. Dan D, Seetahal S, Naraynsingh V. Laparoscopic Heller's myotomy for treatment of achalasia in the Caribbean. West Indian Med J. 2008; 57(5):486-9.

13. Rakita S, Bloomston M, Villadolid D, et al. Age affects presenting symptoms of achalasia and outcomes after myotomy. Am Surg. 2005; 71(5):424-9. Bloomston M, Serafini F, Boyce HW, et al. The "learning curve" in videoscopic Heller myotomy. JSLS. 2002; 6(1):41-7.

14. Bloomston M, Serafini F, Boyce HW, et al. The "learning curve" in videoscopic Heller myotomy. JSLS. 2002; 6(1):417.

15. Dang Y, Mercer D. Treatment of esophageal achalasia with Heller myotomy: Retrospective evaluation of patient satisfaction and disease-specific quality of life. Can J Surg. 2006; 49(4):267-71.

16. Laurino-Neto R, Herbella F, Schlottmann F, et al. Evaluation of esophageal achalasia: From symptoms to the Chicago classification. ABCD Arq Bras Cir Dig. 2018; 31(2):e1376. 